\title{
BLAST Bit Score
}

National Cancer Institute

\section{Source}

National Cancer Institute. BLAST Bit Score. NCI Thesaurus. Code C84318.

A numeric result derived from the BLAST raw score which is normalized to the statistical parameters of the scoring system in use. The BLAST bit score allows for alignment comparisons between independent searches. 